\title{
Care transition from rehabilitation to home: A QI project using the RED Toolkit to decrease readmission rates
}

\author{
Jennifer R. Bernard ${ }^{1}$, Eileen L. Creel ${ }^{* 2}$, Rhonda K. Pecoraro ${ }^{2}$ \\ ${ }^{1}$ Baton Rouge Community College, Baton Rouge, LA, United States \\ ${ }^{2}$ Nursing, Southeastern Louisiana University, Hammond, LA, United States
}

Received: February 3, 2021

DOI: $10.5430 /$ jha.v10n1p46
Accepted: March 8, 2021

Online Published: March 18, 2021

\begin{abstract}
Objective: This quality improvement (QI) project's aim was to lower 30-day healthcare reutilization for patients aged 50 or older with hip fracture using an evidence-based discharge process method, the Re-Engineered Discharge (RED) Toolkit.

Methods: The QI project of a revised patient discharge process to lower healthcare reutilization of Baton Rouge Rehabilitation Hospital (BRRH) hip fracture patients was implemented as an evidence-based quality improvement initiative. Inpatient and outpatient discharge process revisions were implemented at an inpatient rehabilitation facility (IRF) based on Re-Engineered Discharge (RED) Toolkit recommendations. Inpatient revisions included patient barrier identification with associated documentation changes to the IRF interdisciplinary team form. Outpatient modifications consisted of an After-Hospital Care Plan (AHCP), and two post-discharge Telephone Follow-Up (TFU) calls.

Results: Healthcare reutilization and thirty-day hospital readmission for this project were measured at $8.5 \%$ and $5.7 \%$, respectively. A decrease in healthcare reutilization of at least $1.6 \%$ was observed for the IRF. Most participants scored at a high level (88.6\%) of "patient knowledge of self-management" post-intervention. Out of participants who did not attend their first Primary Care Provider (PCP) appointment, 33.3\% experienced healthcare reutilization. This result emphasized the importance of seeing one's PCP post-discharge. Patient satisfaction increased by 5\% and 6.73\%, measured by Hospital Consumer Assessment of HealthCare Providers and Systems (HCAHP) scores for nursing care and physician care, respectively.

Conclusions: Implementation of a RED Toolkit-based discharge process at an IRF positively impacted all three study outcomes and associated healthcare costs in lowering preventable readmissions.
\end{abstract}

Key Words: 30-day readmission, Discharge process, Hip fracture, Inpatient rehabilitation facility, Re-Engineered Discharge Toolkit

\section{INTRODUCTION}

Hospital readmissions result in unfavorable patient outcomes and higher healthcare costs. Measures to lower hospital readmission have been recognized as quality and cost-saving. There are many causes for readmissions, and rates vary widely by facility. ${ }^{[1]}$ Diagnoses for the highest 30 -day readmissions in hospitals are heart failure (27\%), psychoses $(25 \%)$, recent vascular surgery (24\%), chronic obstructive pulmonary disease $(23 \%)$, and pneumonia $(20 \%) .^{[2]}$

Approximately $20 \%$ of all Medicare patients discharged from

*Correspondence: Eileen L. Creel; Email: eileen.creel@ southeastern.edu; Address: 226 Mako Nako Dr. Mandeville, LA. 70471, United States. 
the hospital are readmitted within 30 days. ${ }^{[1-3]}$ The Affordable Care Act's (ACA) Hospital Readmission Reduction Program (HRRP) was established in 2012 to reduce readmission rates through decreasing financial reimbursement to hospitals with high 30-day readmission rates upon discharge..$^{[1,3,4]}$ Excess readmissions are calculated by measuring a hospital's readmission rate and comparing it to national averages. The assessed penalty on readmission is a percent of total Medicare hospital payments and has been increasing by $1 \%$ annually. ${ }^{[1,4]}$

The Medicare Payment Advisory Commission estimated that $12 \%$ of readmissions might be prevented. ${ }^{[5]}$ If even $10 \%$ of patient readmissions were prevented, Medicare could save $\$ 1$ billion in costs. ${ }^{[1]}$ Hip fracture patients have one of the highest cost-saving opportunities compared to other reasons for readmission. Almost $18 \%$ of Medicare pay-for-service hip surgery patients were readmitted within 30 days of discharge. ${ }^{[6]}$ Problems that contribute to preventable readmissions include a lack of standard discharge processes, patients and family members who are poorly prepared for discharge, inadequate medication education, and ineffective communication with post-discharge providers. ${ }^{[3]}$ Medicare payments to inpatient rehabilitation facilities (IRFs) will be lowered if the IRF risk-standardized readmission rates are higher than expected. The Meaningful Measures Initiative was established in October 2017 to lower health care costs and improve patient care. ${ }^{[7]}$ The initiative aims to reduce unnecessary costs, increase efficiencies, and improve the beneficiary experience. The highest priority areas for quality measurement and improvement are identified through the Meaningful Measures to improve patient outcomes. ${ }^{[7]}$ The priority areas of the most significant concern for quality measures are patient and family engagement, patient safety, and clinical processes' effectiveness. ${ }^{[8]}$

Patients are assigned to rehabilitation impairment categories (RICs) under the current IRF prospective payment system. ${ }^{[?]}$ Within each RIC, patients are sorted into case-mix groups (CMGs) based on motor and cognitive level function at admission and discharge. Patients are then further categorized into one of four tiers based on specific comorbidities that increase care costs. In determining appropriate CMG, IRFs assess and score each patient's motor and cognitive function based on the Uniform Data System for Medical Rehabilitation patient assessment instrument, or Functional Independence Measurement (FIM). The FIM score measures a patient's disability level and care burden for a patient's caregivers. ${ }^{[9]}$ Hip fracture patients are often elderly and have a low motor function, reflected in FIM. ${ }^{[10,11]}$ An association was found between lower FIM and higher 30-day readmis- sion among IRF patients compared to patient diagnosis or other variables..$^{[12,13]}$ An assessment of patient FIM has shown particular relevance in identifying healthcare reutilization potential among IRF hip fracture patients.

Federal mandates in 2019 have required that IRFs change from reporting FIM scores to using Improving Medicare Post-Acute Care Transformation Act (IMPACT) scores. A Standardized Post-Acute Care (PAC) Assessment Data Instrument for Quality, Payment, and Discharge Planning will replace the FIM score in IRFs. The new standardized scoring system for patient motor function is also known as the IRF-PAI. ${ }^{[14]}$ Although current literature sources do not yet reflect this change because of recent implementation, PAC scoring through the IMPACT Act's enactment replaces the previous FIM scoring.

A paucity of studies examined reasons for unplanned 30-day readmissions after hip fracture surgery. ${ }^{[6,11]}$ There are limited research studies that have focused on 30-day readmission rates for patients discharged from inpatient rehabilitation facilities (IRFs). High readmission rates among IRFs will soon be financially penalized for acute care hospital readmission according to Medicare policy. ${ }^{[15]}$ The National Quality Forum recently identified 30-day readmission rates as a quality indicator for IRFs. ${ }^{[16]}$ The reduction of healthcare reutilization rates among patients with hip fracture diagnosis in an IRF should be further evaluated to lower healthcare costs and improve patient care quality.

To address readmission, authors of the Re-Engineered Discharge (RED) Toolkit report that a hospital discharge process must be examined in a thorough analysis of established responsibilities and procedures so that functions can be reorganized appropriately. ${ }^{[17]}$ The elements of a successful hospital-based readmission reduction program can be identified by using the RED Toolkit. Implementing the RED Toolkit into practice has provided hospitals with five distinct benefits: A guide to building team relationships, a mechanism to give patient education throughout the hospital stay, common goals between patients and staff, family involvement, enhanced patient learning, and tools which connect patient safety to patient experience. ${ }^{[18]}$

The provision of post-discharge care and telephone follow-up (TFU) have been used as interventions to decrease all-cause 30-day readmission. In a systematic review, study authors Jayakody et al. reported three studies that included TFU and pre-discharge support to patients were shown effective in lowering 30-day readmission. ${ }^{[19]}$ Studies by Lewis et al. and Harrison et al. identified that TFU delivered to older patients after hospital discharge decreased readmission rates 
up to $23.1 \% .^{[20,21]}$ At-risk patients who received TFU were able to accept earlier intervention and support with timely follow-up. ${ }^{[20]}$

A need existed to identify and prevent healthcare reutilization of hip fracture diagnosis in IRFs. The population of patients discharged post-hip fractures was Baton Rouge Rehabilitation Hospital's (BRRH) highest-risk readmission group. A needs assessment for BRRH was performed using SWOT analysis, stakeholder input, and GAP analysis to assess project needs within the IRF.

The GAP analysis showed a failure to identify patient barriers during team conference meetings that may have contributed to readmission. In addition, there was a lack of patientcentered education provided during the patients' stay. The SWOT analysis observed that no revisions had been made to the facility discharge processes or plans directed at lowering 30-day readmission rates. Also, there was uncertainty surrounding the patient's understanding of discharge instructions and knowledge of self-management after discharge to home. Using this information, an evidence-based approach of the RED Toolkit to revise BRRH's discharge processes was implemented to lower healthcare reutilization of hip fracture diagnosis patients.

\section{Objectives}

This QI project's goal was to lower 30-day healthcare reutilization for patients aged $\geq 50$ with hip fracture using an evidence-based discharge process method, the RED Toolkit. Healthcare reutilization encompasses patient hospital readmission, emergency department visits, and urgent care visits. This project's expected outcomes were to lower healthcare reutilization, increase patient knowledge of patient selfmanagement, and increase patient satisfaction in patients with a hip fracture at an IRF. The QI project was to improve health outcomes for an identified population at high risk for healthcare reutilization by following the RED Toolkit protocols, which are inclusive of inpatient discharge processes and post-discharge TFU calls.

Project aims were to decrease healthcare reutilization percentages, increase patient knowledge of self-management, and increase patient satisfaction among IRF hip fracture diagnosis patients. Healthcare reutilization was an outcome measurement used post-RED Toolkit implementation that included hospital readmission, emergency room visits, and urgent care visits. ${ }^{[20]}$

\section{Methods}

The project of a revised patient discharge process to lower healthcare reutilization of BRRH hip fracture patients was implemented as an evidence-based quality improvement initiative. According to Agency for Healthcare Research and Quality (AHRQ), quality improvement is the framework used to improve care delivery to patients. ${ }^{[23]}$ This project is considered a quality process improvement project as it was intended to maintain hip fracture patients' health by reducing IRF healthcare reutilization. Furthermore, improved hospital reutilization percentages indicate improved patient health status and reflect a facility's successful delivery of healthcare.

\subsection{Design and sample}

The project of a revised patient discharge process to lower healthcare reutilization of BRRH hip fracture patients was implemented as an evidence-based quality improvement (QI) initiative. The QI project used a convenience sample of hip fracture patients pending discharge to home. Inclusion criteria consisted of hip fracture patients age $\geq 50$, those discharged to home, those with a caregiver who could provide information if the patient was unable to, and those with a telephone number to be contacted. Exclusion criteria were patients age less than 50, discharged to a long-term care facility, any diagnosis other than hip fracture, patients lacking cognitive comprehension, cognitively impaired patients lacking a caregiver or family member willing to provide information, or those without telephone access.

The sample size was calculated using G*Power 3.1 with the following sample size calculations determined a priori with a chi-square goodness-of-fit test: Effect size $=0.5$; Alpha $=$ 0.05 ; Power $=80 \%$; Sample Size $=32$. The project sample size was 35 out of 45 patients approached. Seven patients refused project participation, expressing physical fatigue and concerns of inadequate family approval for project involvement. An attrition rate of $10 \%$ was factored into account for patients lost to follow-up. Two patients were eliminated from the project due to loss of follow-up on TFU call one. One patient was eliminated due to loss of follow-up on TFU call two. All other participants answered the complete list of script questions for both TFU calls.

\subsection{Outcome measures}

This project measured three outcomes: Healthcare reutilization, patient knowledge of self-management, and patient satisfaction. According to AHRQ, standard measures of 30-day healthcare reutilization are patient percentages with all-cause hospital readmissions within 30 days of discharge, emergency department (ED) visits within 30 days, and urgent care visits within 30 days. The National Quality Forum recently identified 30-day readmission rates as a quality indicator for IRFs. ${ }^{[22]}$ Healthcare reutilization was selected 
as an outcome measure due to its association with lowering of healthcare costs and improved patient care quality in the rehabilitation setting. For this project, outcome one or healthcare reutilization was measured as a percentage or frequency of self-reported patient readmission to a hospital, ED visit, or urgent care center visit. Demographic information was obtained and recorded from the patient's chart, including patient age, race, gender, marital status, medical diagnoses, recent surgeries, and patient IMPACT score. The patient IMPACT score, representing functional motor ability, was used to look for an association between functional physical ability and the percentage of healthcare reutilization.

Patient knowledge of self-management involves the knowledge and ability of a patient to self-manage a medical condition post-discharge. The RED Toolkit employs the term as an outcome measure in evaluating how well patients were taught to take care of themselves upon discharge home. Awareness of how to manage medical conditions is a critical component of TFU. The QI project's second outcome, patient knowledge of self-management, was measured by the percent of patients who could: Correctly state their discharge diagnosis from BRRH, correctly identify the signs and symptoms associated with their BRRH diagnosis, correctly report how to take their medications, accurately provide their PCP's phone number, and report attendance at their first physician appointment post-discharge. ${ }^{[22]}$ Due to its standard usage by AHRQ as a RED Toolkit outcome, patient knowledge of self-management was used in this project as an outcome measurement.

Lastly, the third outcome measured patient satisfaction of their nurses and physicians and if they would recommend BRRH to friends and family. Patient satisfaction is defined by how a patient's expectations about a health encounter were met. The term describes how two people receiving the exact same care may have different expectations for how their healthcare should be delivered. Satisfaction ratings may vary among individual's expectations. The term is used in the RED Toolkit to measure the number of patients replying that nurses always or usually treated them with courtesy and respect, doctors always or usually treated them with courtesy and respect, and would the patient recommend the hospital to friends and family. Following guidelines from the RED Toolkit has been shown to improve overall patient satisfaction with care by improving communication. ${ }^{[22]}$ This outcome was obtained from asking questions used in BRRH's patient satisfaction survey, through patient percentages that reported: 1.) nurses usually treated them with courtesy and respect, 2.) physicians usually treated them with courtesy and respect, and 3.) they would recommend the hospital to friends and family.

Published by Sciedu Press

\subsection{Instrument reliability and validity}

A phone survey based on questions obtained from the RED Toolkit guidelines and three BRRH patient satisfaction questions based on Hospital Consumer Assessment of Healthcare Providers and Systems (HCAHPS) questions were utilized in this project. As an evidence-based method, the RED Toolkit has had significant effects on readmission in randomized controlled trials. A $30 \%$ reduction in hospital reutilization within 30 days of discharge was demonstrated in patients who received the RED Toolkit compared to those receiving usual care. For every seven patients receiving the RED Toolkit, one readmission was prevented. ${ }^{\text {[24] }}$

The HCAHPS survey is the national standard used to measure patient perspective of hospital care that allows valid comparisons across all hospitals. The HCAHPS survey was developed and rigorously tested by AHRQ and endorsed by the National Quality Forum. ${ }^{[25]}$ The study instrument showed validity in its usage of questions from an evidence-based method, the RED Toolkit, which has been used specifically in lowering readmission. The three survey questions adopted from the HCAHPS survey showed validity as part of a proven national standard measuring patient satisfaction. In addition, instrument reliability was demonstrated in using the HCAHPS survey questions, which have been consistently used among various national hospital settings. Questions adapted from the RED Toolkit have shown reliability through usage in AHRQ's Boston clinical trials.

\subsection{Intervention}

A process map was created with input from the project team to analyze and improve BRRH's discharge processes. Mapping showed that BRRH's discharge process began with a BRRH nurse liaison assessing the patient before admission. After the mapping process, the interdisciplinary team created a 24-item checklist of specific barriers to providing a free-text field for patient education. The barrier checklist and education field were added to the interdisciplinary team conference form. These changes were made to BRRH's current discharge processes in a team meeting consensus post-discharge mapping. Delivery of the AHCP and two TFU calls are indicated within beige-colored boxes as part of the RED Toolkit process implementation. (see Figure 1).

Steps taken to implement the RED Toolkit included: Organizing post-discharge outpatient services and medical equipment (step 4); identifying the correct medications and a plan for the patient to obtain them (step 5); teaching a written discharge plan the patient can understand (step 7); assessing the degree of patient understanding of the discharge plan (step 10); expediting transmission of the discharge summary to clinicians accepting care of the patient (step 11); and pro- 
viding telephone reinforcement of the discharge plan (step 12). ${ }^{[17]}$ The BRRH interdisciplinary team designated the case manager as responsible for implementing steps 4, 10, and 11. The patient's discharge nurse ensured that steps 5 and 7 were instituted. The clinical nurse coordinator and unit charge nurse agreed to provide the patients' AHCPs as an addition to step 5 and fulfill TFU as indicated in step 12.

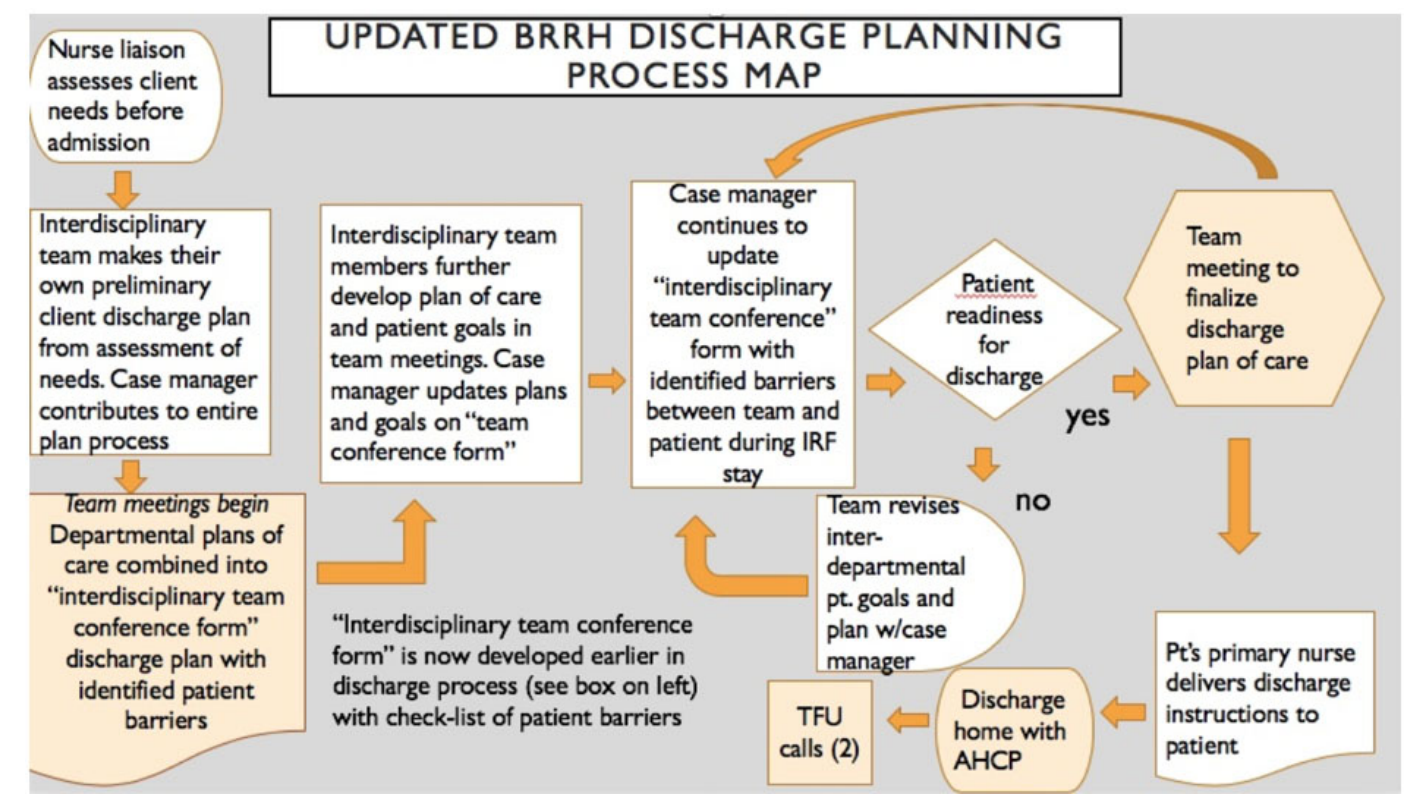

Figure 1. BRRH process map

\subsubsection{TFU training}

Two options for training to conduct follow-up telephone calls were offered to staff. Callers could read the RED Toolkit independently, followed by a review of the contents in a group session, or staff members were shown a series of in-service sessions using the "train-the-trainer" technique. ${ }^{[1]}$ For this project, the clinical nurse coordinator's in-person training for eight TFU sessions was provided.

\subsubsection{AHCP}

An individualized care guide was provided to each project participant prior to discharge. The care guide used was AHRQ's RED Toolkit guide entitled "Taking care of myself: A guide for when I leave the hospital." The care guide identified a patient's: main medical problem, patient medications, allergies, pharmacy location, dietary and exercise recommendations, physician appointments, and how to contact the follow-up physicians. ${ }^{[26]}$

Evidence has shown that hip fracture patients are more likely to be readmitted within 30 days with respiratory issues such as chronic obstructive pulmonary disease (COPD) if one has the condition or may develop pneumonia. ${ }^{[6,10,11]}$ Gardner also found that dehydration was often a factor in the readmission of hip fracture patients. ${ }^{[6]}$ Furthermore, myocardial infarction had a high association with readmission among hip fracture patients. ${ }^{[6,10]}$ Therefore, education handouts from The RED Toolkit emphasizing these high-risk conditions for readmission among hip fracture patients who were most prone to these conditions were provided. Actions were reviewed with patients regarding what to do in these conditions.

\subsubsection{Telephone follow-up}

Two phone calls were made after the patient was discharged home. The first five phone calls were made after week 2 of intervention onset. One phone call was made within 48-72 hours post-discharge, as recommended by The RED Toolkit standards. A scripted consent was read over the phone from an office inside BRRH to remind patients of the project with continued verbal patient consent requested. Physical therapy and all medical/nursing discharge instructions from the patient's chart already provided to the patient by nursing staff were reviewed to identify any specific problem areas needed for follow-up.

The RED Toolkit Discharge Preparation Workbook assisted the caller with a structure to gather all necessary patient information regarding scheduled appointments, diagnoses, and comorbidities before TFU is made. According to AHRQ, the script is only a guide and adapted to the RED Toolkit patient population. ${ }^{[17]}$ The first phone call allowed any questions, misunderstandings, or discharge discrepancies to be addressed by patients or their caregivers. ${ }^{[27]}$ Referrals and 
resources that were patient-specific were noted from the patient's BRRH discharge orders and used to complete the RED Discharge Preparation Workbook. During the first phone call, verification was made that patients who were ordered home health and outpatient physical, occupational, or speech therapy were receiving these services. Follow-up was planned with the BRRH case manager for any apparent service gaps.

The components assessed on the first TFU for each patient, identified from the RED Toolkit, included primary patient diagnosis, symptoms to look out for, any questions about medications, how to obtain the medications, and how to call the PCP or difficulties making scheduled appointments, and any transportation concerns. In this project, planning of the first phone call involved using a guided script and calling the patient between 48 to 72 hours post-discharge, between the hours of 9:00 am and 5:00 pm.

Maintaining communication with one's primary care provider was an important goal of the RED Toolkit. The patient was asked about knowing how to call the primary care provider for any problems and how to call the primary care provider after-hours for any issues. Patients were provided their surgeon or primary care providers' telephone number on the first TFU, as needed. ${ }^{[27]}$

Patients were asked if they have any questions about their medication list during call one. A question in the script from call one also asked about any problems experienced with specific patient barriers related to readmission among BRRH hip fracture patients. This question addressed any identified patient barriers from the revised interdisciplinary team form that could have led to unnecessary readmission.

A second call was made 30 days after discharge to inquire if the patient was readmitted to a hospital, visited an Emergency Department (ED), or visited an urgent care center. The patient was asked whether they attended their first primary care provider (PCP) follow-up appointment. Any unsettling patient symptoms or questions were planned to be managed by telling the patient to call the 911 emergency line for lifethreatening symptoms (such as chest pain or shortness of breath) or telling the patient to call their PCP for other symptoms (example, pain or foot swelling). In addition, questions related to BRRH patient satisfaction were asked during phone call two.

\section{RESUlts}

\subsection{Sample/consenting}

Patients targeted for project participation were BRRH hip fracture adults age 50 or older. A list of potential discharge patients was obtained through a weekly spreadsheet updated and maintained by the facility quality manager. The plan was compiled based on weekly discharge lists provided by department charge nurses. Patients scheduled for discharge were contacted privately in their patient rooms to explain project procedures, including two TFU calls, and to obtain informed consent.

Once consent was obtained, a copy of the After-Hospital Care Plan was provided to the patient prior to discharge. An individualized care plan was created with the patient's discharge medications, primary diagnosis, primary care provider (PCP) phone numbers, and any scheduled appointments. Patient education for any identified home-management barriers was written onto the individual After-Hospital Care Plan (AHCP). All information in the care plan was reviewed with the patient for accuracy. Names of study participants who met the inclusion criteria and consented to involvement were maintained in a locked drawer inside the facility mentor's locked office.

Table 1. Participant demographics $(\mathrm{N}=35)$

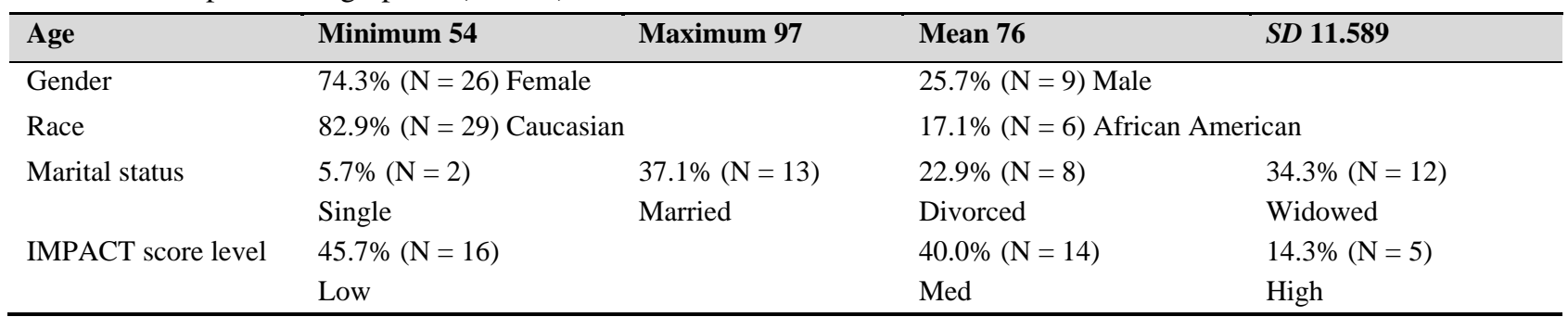

Note. IMPACT= Improving Medicare Post-Acute Care Transformation Act

\subsection{Demographics}

Participant age ranged from 54 to 97 , with mean $M=76$ and standard deviation $\mathrm{SD}=11.589$. Seventy-four percent of project participants were female, and $25.7 \%$ were male. The majority $(82.9 \%)$ of participants were Caucasian, and
17.1\% were African American. Regarding marital status, $5.7 \%$ of participants were single, $37.1 \%$ were married, $22.9 \%$ were divorced, and $34.3 \%$ were widowed. Using the Federal Register (2018) scale of IMPACT levels, $45.7 \%$ of project participants had a level low IMPACT score, $40 \%$ were classi- 
fied as level medium, and $14.3 \%$ had a high IMPACT score. Individual IMPACT scores for this project ranged from 30 to 63 (see Table 1). The former CNO of BRRH estimated 11 hip fracture patients were admitted monthly and discharged to home from the facility. The DNP project was instituted over three months. Therefore, a total sample size of 33 over three months was expected. A project sample size of 35 was attained.

\subsection{Project effect on outcome healthcare reutilization}

The QI project yielded a healthcare reutilization of $8.5 \%$ over the three-month intervention period. At the completion of the quality improvement project, BRRH healthcare reutilization was reduced by $1.6 \%$. Hospital readmission percentages for the United States and Louisiana were $14 \%$ to $16 \%$ for 2015-2019. Project results compared to national, state, and facility readmission percentages are presented (see Table 2).

Table 2. Readmission among National, State, and BRRH data

\begin{tabular}{lllll}
\hline Year & National $^{\mathbf{a}}$ & State $^{\mathbf{a}}$ & BRRH pre-intervention $^{\mathbf{b}}$ & BRRH post-intervention $^{\text {B }}$ \\
\hline $2015-2016$ & $15.8 \%$ & $16.1 \%$ & $10.1 \%$ & -- \\
$2016-2017$ & $15.1 \%$ & $16.2 \%$ & $10.1 \%$ & -- \\
$2017-2018$ & $14.8 \%$ & $15.5 \%$ & $6.5 \%$ & -- \\
2019 & $15.0 \%$ & $15.5 \%$ & N/A & $8.5 \%$ \\
\hline
\end{tabular}

Note. ${ }^{\mathrm{a}}$ United Health Foundation (2019); ${ }^{\mathrm{b}}$ PEPPER (2018)

Table 3. Participants experiencing healthcare reutilization $(\mathrm{N}=3)$

\begin{tabular}{lllllll}
\hline Gender & Age & $\begin{array}{l}\text { Hospital, ED, or } \\
\text { urgent care }\end{array}$ & Reason & $\begin{array}{l}\text { Attended first PCP } \\
\text { appointment }\end{array}$ & $\begin{array}{l}\text { IMPACT } \\
\text { score }\end{array}$ & $\begin{array}{l}\text { IMPACT } \\
\text { score level }\end{array}$ \\
\hline Female & 58 & Hospital & Hip infection & No & 54 & Medium \\
Female & 85 & ED & $\begin{array}{l}\text { Sprained wrist } \\
\text { Pulmonary edema from } \\
\text { chronic heart failure }\end{array}$ & Yes & 37 & Low \\
Male & 90 & Hospital & Low & Low \\
\hline
\end{tabular}

Table 4. Healthcare reutilization and attendance at primary care provider crosstabulation $(\mathrm{N}=35)$

\begin{tabular}{llccc}
\hline \multirow{2}{*}{ Attendance at PCP } & & \multicolumn{2}{c}{ HR } & Total Participants \\
\cline { 3 - 5 } Not attend & Count & No (0) & Yes (1) & 6 \\
& $\%$ in PCP appt & 4 & 2 & $100 \%$ \\
& $\%$ in HR & $66.7 \%$ & $33.3 \%$ & $17.1 \%$ \\
Attended & Count & $12.5 \%$ & $66.7 \%$ & 29 \\
& $\%$ in PCP appt & 28 & $3.4 \%$ & $100 \%$ \\
\multirow{2}{*}{ Total } & $\%$ in HR & $96.6 \%$ & $33.3 \%$ & $32.9 \%$ \\
& Count & $87.5 \%$ & 3 & 35 \\
& $\%$ in PCP appt & 32 & $8.6 \%$ & $100 \%$ \\
\hline
\end{tabular}

Note. $\mathrm{HR}=$ Healthcare Reutilization; $\mathrm{PCP}=$ Primary Care Provider Appointment

There were 32 non-healthcare reutilizations and three healthcare reutilization events in the project. The three participants who experienced healthcare reutilization each had only one event and differed according to age, gender, and type of healthcare reutilization. Two participants $(5.7 \%)$ experienced hospital readmissions. One readmission was related to a postoperative hip infection requiring intravenous antibiotics, and the second readmission was from pulmonary edema related to decompensated chronic heart failure. One participant had an ED visit for a sprained wrist after receiving help from her spouse to move up in bed. Participant reason for healthcare reutilization and IMPACT scores with associated IMPACT levels are provided (see Table 3 ).

Chi-square analysis showed an association was found between healthcare reutilization and attendance at the first PCP 
appointment, with significance $p=.017$. A Cramer's V significance of $p=.017$ confirmed the association as strong between the two variables (see Tables 4 and 5). There was no association between healthcare reutilization and: IMPACT score level $(p=.147)$, knowing the signs and symptoms to be concerned about with one's diagnosis $(p=.971)$, knowing one's PCP phone number $(p=.365)$, patient knowledge of self-management $(p=.408)$, or patient satisfaction $(p=$ $.567)$.

Table 5. Chi-square test and Cramer's V test strength association $(\mathrm{N}=35)$

\begin{tabular}{lccc}
\hline & value & df & sig \\
\hline Pearson Chi-square & $5.666^{\mathrm{a}}$ & 1 & .017 \\
Continuity Correction & 2.494 & 1 & .114 \\
Likelihood Ratio & 4.138 & 1 & .042 \\
Linear by linear association & 5.504 & 1 & .019 \\
Cramer's V & .402 & -- & .017 \\
N of Valid cases & 35 & -- & -- \\
\hline
\end{tabular}

Note. $\mathrm{df}=$ degrees of; $\operatorname{sig}=$ significance 2 -sided with $p<.05$; ${ }^{2} 2$ cells $(50.0 \%)$ have expected count less than 5 ; The minimum expected count is .51

\subsection{Project effect on outcome "Patient Knowledge of Self-Management"}

A primary objective of The RED Toolkit was to teach patients how to take care of themselves after they were discharged home. ${ }^{[17]}$ The RED Toolkit's knowledge outcome measures included the percentage of patients who reported an increase in healthcare knowledge, as follows: 1) the percentage who correctly reported during the post-discharge TFU call the reason for their hospital visit $=100 \% ; 2$ ) the percentage who correctly reported during the post-discharge TFU call the symptoms to watch out for or how to manage their condition $=66 \% ; 3$ ) the percentage who correctly reported during the post-discharge TFU call how to take their medicines $=100 \%$; 4) the percentage who knew their primary care provider's phone number $=80 \%$; and 5 ) the percentage who attended their first PCP appointment $=83 \%$. Three studies were used as pre-intervention comparison data. ${ }^{[28-30]}$ A majority of patients $(91 \%)$ reported no questions about their BRRH identified patient barrier.

Table 6. "Patient knowledge of self-management" level $(\mathrm{N}=35)$

\begin{tabular}{lll}
\hline Participant Knowledge level & Frequency & Percent \\
\hline Low & 1 & $2.9 \%$ \\
Medium & 3 & $8.5 \%$ \\
High & 31 & $88.6 \%$ \\
\hline
\end{tabular}

As listed above, five outcome measures comprised the outcome 'patient knowledge of self-management.' One point was provided for each question answered correctly, producing an outcome score between one and five. Next, participant total scores were assigned three levels from the points earned on the five TFU script questions answered correctly: 1-2 = Low level; 3 = Medium level; 4-5 = High level. The low, medium, and high levels of "patient knowledge of selfmanagement" are provided for all participants. A majority of participants $(88.6 \%)$ scored at a high level for outcome "patient knowledge of self-management" (see Table 6). Patient feedback indicated that BRRH nurses were excellent at providing medication education at discharge.

Table 7. BRRH savings

\begin{tabular}{|c|c|c|c|c|c|c|}
\hline & \multirow{2}{*}{$\begin{array}{l}\text { Annual Quantity } \\
\text { Total pts }(\mathrm{N}=794)\end{array}$} & \multicolumn{2}{|c|}{ Annual Readmission \% } & \multicolumn{2}{|c|}{ Total Annual Lost Revenue } & \multirow{2}{*}{$\begin{array}{l}\text { Total Annual savings } \\
\text { (minus } \$ 1,389 \text { cost) }\end{array}$} \\
\hline & & $12 \%$ & $6 \%$ & $12 \%$ & $6 \%$ & \\
\hline $\begin{array}{l}\text { Hip fracture IRF patients } \\
\text { CMS readmission penalty: } \\
\$ 455.49 / \text { patient }\end{array}$ & $\begin{array}{l}132 \text { hip fracture } \\
\text { patients }\end{array}$ & $\begin{array}{c}16 \text { readmitted } \\
\text { patients }\end{array}$ & $\begin{array}{l}8 \text { readmitted } \\
\text { patients }\end{array}$ & $\$ 7,287$ & $\$ 0$ & $\$ 5,898$ \\
\hline $\begin{array}{l}\text { All discharged IRF patients } \\
\text { CMS readmission penalty: } \\
\text { \$445.08/patient }\end{array}$ & $\begin{array}{l}662 \text { all other } \\
\text { patients }\end{array}$ & $\begin{array}{c}79 \\
\text { readmitted } \\
\text { patients }\end{array}$ & $\begin{array}{l}40 \\
\text { readmitted } \\
\text { patients }\end{array}$ & $\$ 35,161$ & $\$ 0$ & $\$ 35,161$ \\
\hline Total Annual Savings & & & & & & $\$ 41,059$ \\
\hline
\end{tabular}

\subsection{Project effect on outcome "Patient Satisfaction"}

There was an increase in HCAHP scores for questions one and two after project implementation. Patient satisfaction for nursing care (question one) increased by $5 \%$, and patient satisfaction with physician care (question two) increased by $6.73 \%$. No change in scoring for question three, the recommendation of BRRH to friends or family, was noted.

\subsection{Project savings}

The total Medicare payment to a facility for a hip fracture stay is $\$ 15,183$ and for all medical conditions is $\$ 14,836 .^{[31]}$ Payments reduced per patient can amount to as much as $3 \%$ of the total cost of patient reimbursement based on patient readmission percentages exceeding set standards. ${ }^{[8]}$ If a $3 \%$ CMS penalty for excessive readmission is applied to 
BRRH patients at $12 \%$ readmission, the penalty cost would be $\$ 455.49$ per hip fracture patient and $\$ 445.08$ per patient for all other medical conditions. There was an average of 66 monthly patient discharges at BRRH in 2019 for an annual total of 794 discharged patients (A. Landry, personal communication, January 27,2020 ). A $6 \%$ readmission was the project aim, which would result in no penalty assessed. The savings to BRRH based on $12 \%$ annual readmission compared to $6 \%$ BRRH goal readmission is presented in Table 7.

\section{Discussion}

Project results were most similar to findings from Kumar et al., which showed a greater association of readmission among IRF females than males, in addition to a lower discharge motor function score. ${ }^{[32]}$ Results were consistent with the findings of four study authors ${ }^{[10,12,16,33]}$ who identified high-risk group IRF patients for 30-day readmission to be of older age and with lower motor function scores. However, these studies showed an increased risk of 30-day readmission for males over females, which was not reflected in this project.

The project decreased healthcare reutilization and hospitalization by $1.6 \%$ and $5.4 \%$, respectively. Project findings were consistent with results in Mitchell et al., which noted that usage of the RED Toolkit with patient-tailored discharge processes, including TFU calls, demonstrated an all-cause 30 -day readmission reduction. ${ }^{[18]}$ Hospitals that were successful in using the RED Toolkit implementation in discharge process revisions had redesigned their work processes in a multidisciplinary approach. ${ }^{[18]}$ Similar to Harrison et al., study authors found that receiving a discharge phone call was associated with lower readmission rates. ${ }^{[21]}$ Jayakody et al. reported that 5 out of 10 studies that used TFU with other interventions lowered readmission. ${ }^{[19]}$ In addition, Lewis et al. noted that early telephone calls post-discharge could lower readmission. ${ }^{[20]}$

No association was noted between healthcare reutilization and IMPACT scores or levels. These results were in contrast to six study authors ${ }^{[10,12,13,16,32,33]}$ who noted that patient functional status (FIM or other mobility score) impacted 30-day readmission rates. The lack of association between healthcare reutilization and IMPACT score could be due to a small project sample size of 35 , compared to larger IRF studies of aggregate data consisting of over 100,000 patients. Project replication is recommended to other IRFs using a greater sample size.

One participant experienced hospital readmission due to pulmonary edema secondary to chronic heart failure. This is similar to findings from Galloway et al., noting that heart failure was one of the most common diagnoses for IRF readmission. ${ }^{[33]}$ Shivok-Jefferson reported that heart failure was a major reason for patient readmission from a skilled rehabilitation facility. ${ }^{[34]}$ In addition, study authors Kates et al. and Martin et al. observed that pulmonary causes for readmission were most often identified in the 30-day readmission of hospitalized hip fracture patients. ${ }^{[10,11]}$

A significance of $p=.017$ was shown between healthcare reutilization and attendance at one's first PCP appointment. Out of participants who attended their first PCP appointment, $3.4 \%$ experienced healthcare reutilization. Out of participants who did not attend their first PCP appointment, 33.3\% experienced healthcare reutilization. This result emphasized the importance of seeing one's PCP post-discharge. In accordance with these findings, one study reported that $40 \%$ of a sample comprised only of readmitted patients did not see their PCP following their initial discharge. ${ }^{[34]}$

In this project, three TFU questions measured patient knowledge of self-management between $91 \%-100 \%$. Patient satisfaction was measured at $94.6 \%, 97.3 \%$, and $100 \%$ among the three survey questions to measure this outcome. Similar to RED Toolkit-based findings from Osei, data obtained from three TFU questions measuring patient knowledge of self-management showed that study outcomes ranged between $92 \%-100 \% .{ }^{[35]}$ Patient satisfaction was shown to be at 97\% post-implementation with the usage of survey questions recommended by the RED Toolkit. ${ }^{[35]}$

Measurement of post-intervention outcome "patient knowledge of self-management" was substantially higher than all seven comparator baseline measures except for attendance at one's first PCP appointment, which was only 3\% lower than the comparator. A significance of $p=.002$ was observed between outcome "patient knowledge of self-management" and outcome "patient satisfaction". Patient satisfaction HCAHP scores for nursing and physician care increased by $5 \%$ and $6.73 \%$, respectively. These results indicate that knowing how to take care of oneself may lead to greater patient satisfaction. Similar findings were observed in Nicholson et al., where interventions that improved patient knowledge were directly linked to increased patient satisfaction with their care. ${ }^{[31]}$

Study participants reported that they appreciated the communication from project staff about their post-discharge scheduled PCP appointments. In alignment with this result, findings from a study focused on improving patient communication by Kemp et al. showed that if patients had more involvement in their care decisions and receipt of written discharge instructions, unplanned hospital readmissions would be reduced up to one-year post-discharge. ${ }^{[36]}$ According to 
the RED Toolkit authors, following the RED Toolkit guidelines have been shown to improve overall patient satisfaction with care received by improving communication. ${ }^{[22]}$

\subsection{Limitations}

Limitations included a small sample size of patients. Sudden drops in BRRH patient census hindered project recruitment efforts. According to AHRQ, RED Toolkit implementation can take between 6 to 12 months or longer due to specific processes at the clinical site. ${ }^{[22]}$ Therefore, a more significant project effect may have been shown during a longer implementation period to acquire a larger sample size and to adhere with AHRQ timeframe recommendations. A staff change among clinical nurse coordinators occurred during implementation, with processes slowed to allow for the nurse's adjustment to her newly acquired position. In addition, there was an inability to use BRRH baseline data for 'patient knowledge of self-management' because no prior data existed for this outcome. Instead, previous studies were used as comparison data to post-intervention findings.

\section{Conclusions}

In conclusion, a positive effect was observed from three project outcomes based on a revised discharge process implemented at BRRH. The project results indicated that a RED Toolkit-based discharge process positively impacted inpatient and outpatient discharge components at an IRF. Results showed that overall patient satisfaction at BRRH improved, possibly due to an increase in knowledge of self-management. Therefore, one implication is for staff to improve their communication with patients, to increase patient awareness of their scheduled PCP and other appointments earlier in the discharge process. Improving communication may further increase patient knowledge of self-management, with an associated increase in patient satisfaction.

The increased percentage of healthcare reutilization among patients who did not attend their PCP appointment underscores a need to reinforce the importance of attending one's initial PCP appointment. Also, identifying patient barriers and providing barrier education throughout the discharge process could improve patient self-management of their home care. Overall, the project resulted in an improvement in patient quality outcomes in an IRF setting. Financial savings were also obtained by lowering preventable 30-day readmission to the facility and national healthcare system.

\section{ACKNOWLEDgements}

Mr. Derrick Landreneau, the former CNO of BRRH, allowed this QI project to be implemented due to his unwavering commitment to quality rehabilitative care. After his departure, project continuation was only possible due to Ms. Laura Simon's expert logistical support that she effortlessly provided to the study authors. The authors express their deepest gratitude to BRRH administration and staff.

\section{ETHICS}

The Institutional Review Board (IRB) of Baton Rouge General Medical Center (BRGMC) and Southeastern Louisiana University Institutional Review Board (IRB) approval was granted prior to study initiation with IRB Number 2020-002. Subjects were protected from the risk as much as possible by a complete explanation of the RED Toolkit-based discharge process at the time of consent.

\section{CONFLICTS OF INTEREST Disclosure}

The authors declare they have no conflicts of interest.

\section{REFERENCES}

[1] McIlvennan CK, Eapen ZJ, Allen LA. Hospital readmissions reduction program. Circulation. 2015; 131(20): 1796-1803. PMid: 25986448. https://doi.org/10.1161/CIRCULATIONAHA. 114 .010270

[2] Kripalani S, Theobald CN, Anctil B, et al. Reducing hospital readmission: Current strategies and future directions. Ann Rev Med. 2015; 65: 471-475. PMid: 24160939. https://doi.org/10.1146/an nurev-med-022613-090415

[3] Nelson JM, Rosenthal L. How nurses can reduce hospital readmissions. [Internet]. 2015 May. Available from: https://www.myamericannurse. com/nurses-can-hel p-reduce-hospital-readmissions/

[4] Centers for Medicare \& Medicaid Services. Inpatient facility PPS: Data files. [Internet]. 2018 Oct. Available from: https://www.cms.gov/Medicare/Medicare-Fee-for-Ser vice-Payment/InpatientRehabFacPPS/Data-Files.html

Published by Sciedu Press
[5] Medicare Payment Advisory Commission. Mandated report: The effects of the hospital readmissions reduction program. [Internet]. 2018 June. Available from: http://www.medpac.gov/docs/default-source/reports/ jun18_ch1_medpacreport_appendix_sec.pdf?sfvrsn=0

[6] Gardner K. Hip fractures: What information does the evidence show that patients and families need to decrease 30-day readmission? Orthop Nurs. 2015; 34(6): 324-331. PMid: 26575502. https://doi.org/10.1097/NOR.0000000000000190

[7] Federal Register. Medicare program; Inpatient Rehabilitation Facility Prospective Payment System for federal fiscal year 2019. [Internet]. 2018 May. Available from: https://www.federalregister.go $\mathrm{v} /$ documents/2018/05/08/2018-08961/medicare-program -inpatient-rehabilitation-facility-prospective-pay ment-system-for-federal-fiscal

[8] Centers for Medicare \& Medicaid Services. Clinical quality measures basics [Internet]. 2019 Oct. Available from: https: 
//www.cms.gov/Regulations-and-Guidance/Legislation/ EHRIncentivePrograms/ClinicalQualityMeasures.html

[9] Medicare Payment Advisory Commission. MedPAC comment on CMS's proposed rule on the Inpatient Rehabilitation Facility PPS for FY 2020. [Internet]. 2019 June. Available from: http://www .medp ac.gov/docs/default-source/comment-letters/0614201 9_irf_fy2020_medpac_comment_v2_sec.pdf?sf vrsn $=0$

[10] Kates SL, Behrend C, Mendelson DA, et al. Hospital readmission after hip fracture. Arch Orthop Trauma Surg. 2014; 135(3): 32937. PMid: 25550095. https ://doi .org/10.1007/s00402-014 $-2141-2$

[11] Martin CT, Gao Y, Pugely AJ. Incidence and risk factors for 30-day readmissions after hip fracture surgery. Iowa Orthop J. 2016; 36: 155-160. Available from: https ://www.ncbi.nlm.nih.gov/pmc /articles/PMC4910810/

[12] Fisher SR, Graham JE, Krishnan S, et al. Predictors of 30-day readmission following inpatient rehabilitation for patients at high risk for hospital readmission. Phys Ther. 2016; 96(1): 62-70. PMid 26359271. https://doi.org/10.2522/ptj. 20150034

[13] Shih SL, Zafonte R, Bates DW, et al. Functional status outperforms comorbidities as a predictor of 30-day acute care readmissions in the inpatient rehabilitation population. J Am Med Dir Assoc. 2016; 17 921-926. PMid: 27424092. https://doi.org/10.1016/j.jamd a. 2016.06 .003

[14] Federal Register. Medicare program; Inpatient Rehabilitation Facility Prospective Payment System for federal fiscal year 2016 [Internet] 2015 Aug. Available from: https://www.federalregister.go v/documents/2015/08/06/2015-18973/medicare-program -inpatient-rehabilitation-facility-prospective-pay ment-system-for-federal-fiscal\#h-84

[15] Centers for Medicare \& Medicaid Services. Hospital readmissions reduction program (HRRP). [Internet]. 2018 Sept. Available from: https://www.cms.gov/medicare/quality-initiatives-p atient-assessment-instruments/value-based-program $\mathrm{s} / \mathrm{hrrp} /$ hospital-readmission-reduction-program.html

[16] Ramey L, Goldstein R, Zafonte R, et al. Variation in 30-day readmission rates among medically complex patients at inpatient rehabilitation facilities and contributing factors. J Am Med Dir Assoc. 2016; 17: 730-736. PMid: 27161849. https ://doi.org/10.101 $6 / j \cdot j$ amda. 2016.03 .019

[17] Agency for Healthcare Research and Quality. Re-Engineered Discharge (RED) toolkit [Internet]. $2020 \mathrm{Feb}$. Available from: https://www . ahrq.gov/professionals/systems/hospita l/red/toolkit/index.html

[18] Mitchell SE, Martin J, Holmes S, et al. How hospitals reengineer their discharge processes to reduce readmissions. J Healthc Qual 2016; 38(2): 116-126. PMid: 26042743. https ://doi .org/10.1 097/JHQ.0000000000000005

[19] Jayakody A, Bryant J, Carey M, et al. Effectiveness of interventions utilizing telephone follow up in reducing hospital readmission within 30 days for individuals with chronic disease: A systematic review. BMC Heal Ser Res. 2016; 16(1): 1-9. PMid: 27538884. https://doi.org/10.1186/s12913-016-1650-9

[20] Lewis E, Sampari S, Boyd-Skinner C. Telephone follow up calls for older patients after hospital discharge. Age Ageing. 2017; 46: 544-546. PMid: 28104599. https://doi.org/10.1093/ageing /afw251

[21] Harrison PL, Hara PA, Pope JE, et al. The impact of post discharge telephonic follow-up on hospital readmissions. Pop Health Manag. 2011; 14(1): 27-32. PMid: 21090991. https://doi .org/10.108 9/pop.2009.0076
[22] Agency for Healthcare Research and Quality. Tool 6: How to monitor RED implementation and outcomes. [Internet] 2013 Mar. Available from: https://www.ahrq.gov/patient-safety/settings/h ospital/hai/red/toolkit/redtool6.html

[23] Agency for Healthcare Research and Quality. Module 4: Approaches to quality improvement [Internet]. 2013 May. Available from: https : //www.ahrq.gov/ncepcr/tools/pf-handbook/mod4.html

[24] Agency for Healthcare Research and Quality. Tool 1: Overview. [Internet]. 2013 Mar. Available from: https://www.ahrq.gov/hai /red/toolkit/redtool1.html

[25] Centers for Medicare \& Medicaid Services. HCAHPS Fact Sheet. [Internet] 2017 Oct. Available from: https://hcahpsonline.org/globalassets/hcahps/f acts/hcahps_fact_sheet_november_2017.pdf

[26] Agency for Healthcare Research and Quality. Taking care of myself: A guide for when I leave the hospital [Internet]. 2020 Nov. Available from: https://www.ahrq.gov/questions/resources/going -home/index.html

[27] Agency for Healthcare Research and Quality. How to conduct a post-discharge follow-up phone call. [Internet]. 2013 Mar. Available from: https://www.ahrq.gov/patient-safety/settings/h ospital/hai/red/toolkit/redtool5.html

[28] American Association of Retired Persons. Beyond 50.09 chronic care: A call to action for health reform. [Internet]. 2009. Available from: https://assets .aarp.org/rgcenter/health/beyond _50_hcr_1.pdf

[29] Kambli S. Fractured patient's knowledge regarding care and treatment. Int J Sci Res. 2014; 3(7): 1178-1181.

[30] Nicholson TE, Edwards L, McArdle P. Knowledge is power: A quality improvement project to increase patient understanding of their hospital stay. BMJ Qual Improv Rep. [Internet]. 2017; 6(1): 1-6. PMid: 28321297. https ://doi .org/10.1136/bmjquality .u207103.w3042

[31] Center for Medicare Advocacy. Inpatient Rehabilitation Facilities and Skilled Nursing Facilities: Vive La Difference! 2014 July [Internet]. Available from: https://medicareadvocacy.org/i npatient-rehabilitation-facilities-and-skilled-nur sing-facilities-vive-la-difference/

[32] Kumar A, Karmarkar AM, Graham JE, et al. Comorbidity indices versus function as potential predictors of 30-day readmission in older patients following post-acute rehabilitation. J Gerontol A Biol Sci Med Sci. 2016; 72(2): 223-228. PMid: 27492451. https://doi.org/10.1093/gerona/glw148

[33] Galloway RV, Karmakar AM, Graham JE, et al. Hospital readmission following discharge from inpatient rehabilitation for older adults with debility. Phys Ther. 2016; 96(2): 241-251. PMid: 26637650. https://doi.org/10.2522/ptj. 20150030

[34] Shivok-Jefferson T. Factors that may contribute to unnecessary hospital readmissions of elderly patients within 30 days of discharge from a skilled rehabilitation facility [dissertation]. [Center Valley (PA)]: DeSales University; 2016.

[35] Oesi M. The impact of telephone follow-up calls on 30-day readmissions in older adults post total joint replacement surgery [dissertation]. [Irvine (CA)]: Brandman University; 2017.

[36] Kemp KA, Quan H, Santana MJ. Lack of patient involvement in care decisions and not receiving written discharge instructions are associated with unplanned readmissions up to one year. Patient Exp J. 2017; 4(2): 13-22. Available from: https ://pxjournal .org/journal/ vol4/iss2/4https://doi.org/10.35680/2372-0247.1205 